\title{
Caracterización de la crianza de trucha arcoíris (Oncorhynchus mykiss) en la provincia de Chincheros, Apurímac, Perú
}

\author{
Characterization of the rainbow trout (Oncorhynchus mykiss) breeding in the \\ province of Chincheros, Apurimac, Peru
}

Ivar Zárate M. ${ }^{1,4}$, Cristian Sánchez P. ${ }^{2}$, Hugo Palomino C. ${ }^{3}$, Carlos Smith D. ${ }^{2}$

\section{Resumen}

\begin{abstract}
El estudio tuvo como finalidad caracterizar la producción de trucha arcoíris (Oncorhynchus mykiss) en la provincia de Chincheros, Apurímac. Se entrevistó al 85\% de productores $(n=21)$ mediante una encuesta entre noviembre y diciembre de 2017. El $85.7 \%$ de los encuestados fueron mayores de 40 años, donde el $42.9 \%$ contaba con educación secundaria completa. Las dos principales actividades desarrolladas por los productores fue la agricultura $(38.0 \%)$ y la crianza de truchas $(33.3 \%)$. Más del $50 \%$ producía arriba de tres toneladas de trucha al año. El 38.0\% contaba con pozas de tierra y cemento, el abastecimiento de agua fue principalmente de manantial (85.7\%). Alrededor de la mitad de los encuestados contaba con servicios de agua potable y electricidad en su centro productivo. La mayoría compraba alevines como semilla (85.7\%), que en su mayoría son criados durante 7 y 8 meses. Los mayores episodios de mortalidad se dan en la estación de verano ( $85 \%$ ) y son atribuidos a los cambios en la temperatura y al descenso del oxígeno. El $75 \%$ de los productores utiliza alimento balanceado y la mayoría lo racionan tres veces al día. El peso de venta de los peces fue entre 200 a $250 \mathrm{~g}$, los cuales son vendidos principalmente como trucha entera a un precio entre 15 y 25 soles por kilogramo (1 US\$=S/.3.25).
\end{abstract}

Palabras clave: trucha arcoíris; Chincheros; productores

\footnotetext{
${ }^{1}$ Municipalidad Provincial de Chincheros, Apurímac, Perú

${ }^{2}$ AQUA Consultorías y Servicios (AQUA CYS), Lima, Perú

${ }^{3}$ Dirección Sub regional de Producción Andahuaylas, Apurímac, Perú

${ }^{4}$ E-mail: Ivar.zarate@upch.pe
}

Recibido: 7 de marzo de 2018

Aceptado para publicación: 12 de septiembre de 2018 
The purpose of the study was to characterize the production of rainbow trout (Oncorhynchus mykiss) in the province of Chincheros, Apurímac. Interviews were done to $85 \%$ of the producers $(\mathrm{n}=21)$ through a survey between November and December 2017. In general, $85.7 \%$ of the respondents were over 40 years old, where $42.9 \%$ had complete secondary education. The two main activities developed by the producers were agriculture (38.0\%) and trout farming (33.3\%). More than $50 \%$ produced more than three tons of trout per year. The pools were constructed with soil and cement (38.0\%), the water supply was mainly from springs $(85.7 \%)$. About half of the respondents had potable water and electricity services in their productive centres. Most purchase fingerlings as seed $(85.7 \%)$, which are mostly bred for 7 and 8 months. Most episodes of mortality occur in the summer season $(85 \%)$ and are attributed to changes in temperature and oxygen descent. Moreover, $75 \%$ of the producers use balanced food and the majority ration it three times a day. The weight of sale was between 200 to $250 \mathrm{~g}$, which are sold mainly as whole trout at a price of between 15 and 25 soles per kilogram ( $1 \mathrm{US} \$=\mathrm{S} / .3 .25$ ).

Key words: rainbow trout; Chincheros; producers

\section{INTRODUCCIÓN}

La acuicultura mundial crece de manera sostenida, habiendo llegado a los 74 millones de toneladas de producción en 2014 (FAO, 2016); cifra que representa cerca del $50 \%$ del total de productos hidrobiológicos consumidos. Esta producción equipara a la pesca y perfila a la acuicultura como la actividad productora de alimentos con el mayor potencial para satisfacer la seguridad alimentaria mundial (FAO, 2017), diversificando la oferta económica de países en desarrollo, mejorando la calidad de vida de productores en comunidades rurales, y brindando empleo a personas involucradas en los demás eslabones productivos (García et al., 2013).

La acuicultura en el Perú es una actividad que viene mostrando un acelerado incremento en la producción en los últimos años, alcanzando las 93.4 toneladas métricas (TM) en 2016; siendo la trucha (Oncorhynchus mykiss) la principal especie acuícola que ha generado este crecimiento con $40946 \mathrm{TM}$ (PRODUCE, 2016). Este crecimiento está sostenido por la gran cantidad de recursos hídricos identificados como apropiados para esta actividad, especialmente en las zonas altoandinas (PRODUCE, 2017). Sin embargo, las condiciones ambientales favorables para el cultivo de la trucha no garantizan el éxito, el cual está relacionado también con un mercado que acepte el producto y políticas públicas que permitan el fomento de esta actividad (García et al., 2013).

En el Perú, la producción y comercialización de la trucha en las zonas altoandinas se caracteriza por el empirismo y las dificultades que esto conlleva (Yapuchura, 2002), La región Apurímac cuenta con un área de 12500 ha de recursos hídricos con potencial para la producción de truchas, que ha permitido una producción de solo 104 TM en 2015 (PRODUCE, 2016), de allí la importancia de caracterizar los sistemas de producción para hacerlos más eficientes (Zaldívar, 1989). Para este fin, se puede utilizar un diagnóstico situacional a través de encuestas de una sola visita o múltiples visitas (Ruíz, 1989). El presente trabajo de investigación tuvo como objetivo caracterizar la producción de truchas de la provincia de Chincheros, región Apurímac, identificando sus limitaciones y potencialidades. 


\section{Materiales y Métodos}

El estudio se llevó a cabo en la provincia de Chincheros, ubicada a 2795 msnm en el departamento de Apurímac, Perú. Inicialmente, se realizaron entrevistas a las autoridades locales con la finalidad de identificar y cuantificar a los productores de trucha arcoíris (Oncorhynchus mykiss). Posteriormente, se elaboró una encuesta de 27 preguntas, la cual fue validada por tres especialistas del campo acuícola.

Las encuestas para la recolección de datos se hicieron con base a una única visita. Se logró encuestar a 21 acuicultores, que representan el $85 \%$ de los productores de trucha de la provincia. Los resultados fueron analizados mediante estadística descriptiva.

\section{Resultados y Discusión}

\section{Productor}

Los 21 encuestados registraron una edad media de $46.5 \pm 10.7$ años, siendo el $85.7 \%$ mayores de 40 años. El 19\% de los encuestados fueron mujeres. La mayoría de los productores contaban con secundaria completa $(42.9 \%)$, seguido por los que tenían primaria completa $(14.3 \%)$, superior técnico $(14.3 \%)$ y educación universitaria $(9.5 \%)$, mientras que los productores que no completaron la primaria y aquellos que no culminaron la secundaria fueron $9.5 \%$. El $52.4 \%$ expresaron que contaban con agua potable y el $57.1 \%$ con electricidad en su centro productivo. Los productores que contaban simultáneamente con agua potable y electricidad representaron el $47.6 \%$.

El 33.3\% de los productores se dedicaban en forma exclusiva a la crianza de truchas, mientras que $38 \%$ realizan la agricultura como actividad principal. Así mismo, el $9.5 \%$ realizaban ambas actividades, mientras que el $19.2 \%$ realizaba algún otro tipo de ac- tividad adicional. El $42.9 \%$ de los productores manifestaron que tenían una producción anual menor a las tres toneladas métricas.

\section{Manejo}

El tiempo que dedican los productores a la actividad piscícola varía desde los que se toman solo una hora (4.8\%), entre 2 y 3 horas $(9.5 \%)$, entre 3 y 4 horas $(23.8 \%)$, entre 4 y 5 horas $(38.0 \%)$ y los que dedican más de 5 horas al día (23.9\%).

Las pozas de cultivo fueron en su mayoría de tierra y cemento $(38.0 \%)$, seguido por los estanques de tierra (23.9\%), cemento y tierra $(19.0 \%)$, pozas de cemento $(14.3 \%)$ y de piedra $(4.8 \%)$. Según FONDEPES (2014), los estanques de tierra, si bien son de bajo costo, ocasionan problemas de sólidos en suspensión, dificultades de limpieza y de desinfección, y dependiendo del tipo de tierra pueden presentar diversos grados de pérdida de agua por filtración (Speare et al., 1995; Ayik y Akyurt, 1996).

Las producciones que se abastecen de agua de manantial representan el $85.7 \%$, mientras que el $14.3 \%$ se abastece con agua de río y manantial.

El $85.7 \%$ de los encuestados manifestó que compra alevines y el $14.3 \%$ adquieren ovas embrionadas. Estas provienen de Chincheros (38.1\%), Andahuaylas (23.8\%), Uripa $(23.8 \%)$ y de Estados Unidos de Norteamérica (14.3\%).

Los encuestados expresaron que el tiempo de cría oscila entre 7 y 8 meses $(47.6 \%)$, seguido por los que crían por 5 y 6 meses (28.6\%) y los que lo hacen entre 8 y 12 meses $(23.8 \%)$. Solo uno de los encuestados refirió conocer la densidad de su cultivo.

La utilización de registros de producción es indispensable para una adecuada producción (FONDEPES, 2014); sin embargo, el $70 \%$ de los productores manifestaron no llevar este tipo de registros. 


\section{Sanidad}

El $85.7 \%$ de los productores refieren que registran episodios de mortalidad, principalmente durante el periodo de estiaje, mientras que solo el $14.3 \%$ lo tiene durante la época de lluvias. La mayor mortalidad en estiaje puede deberse al bajo caudal del agua que ingresa a los centros de cultivo durante esa época del año (FONDEPES, 2014). En este sentido, Davidson et al. (2011) y Ruyet et al. (2008) indican que se requiere un flujo constante de agua a lo largo del año para asegurar la producción.

Los productores asocian las causas de mortalidad con la temperatura del agua (47.5\%), la temperatura asociada a la caída de oxígeno (4.8\%), la temperatura asociada con la escasez de agua (4.8\%), la escasez de agua (19.0\%), al agua asociada con la mala semilla (4.8\%), a enfermedades $(9.5 \%)$, al alimento (4.8\%) y a fenómenos climatológicos $(4.8 \%)$. Para la prevención de enfermedades, el $23.8 \%$ manifiesta que utiliza desinfectantes, el $4.8 \%$ utiliza sal, el $66.6 \%$ utiliza ambos elementos y el $4.8 \%$ usa cal.

\section{Alimentación}

Más del $75 \%$ de productores hacen uso de alimento comercial, $14.3 \%$ mezcla el alimento comercial con alimento elaborado por ellos, y 9.7\% mezcla con arroz o alimento para pollos. Un tercio de los encuestados manifiesta que alimenta con una frecuencia de tres veces al día, $28.6 \%$ alimenta dos veces al día y el restante solo lo hace una vez al día. Al respecto, FONDEPES (2014) señala que la frecuencia de la alimentación varía a lo largo de la etapa productiva, siendo entre 2 y 4 veces al día la frecuencia adecuada durante la etapa de engorde.

Dos tercios de los encuestados refieren que toman la temperatura del agua antes de alimentar a los peces. La medición de este parámetro es necesario para estimar la cantidad de alimento que se suministrará diaria- mente (Becker et al., 2005) Por otra parte, el $66.7 \%$ manifestó que realiza el almacenamiento del alimento en sus propias viviendas, $23.8 \%$ lo hace en almacenes exclusivos y 9.5\% lo almacena al aire libre. FONDEPES (2014) indica que el almacén para el alimento es de suma importancia, por lo que debe ser diseñado y construido de manera que esté protegidos del sol, humedad, insectos y roedores.

\section{Comercialización}

La cosecha se realiza mayoritariamente cuando las truchas alcanzan pesos entre 200 a $250 \mathrm{~g}(71.4 \%)$, seguido por pesos entre 250 y $350 \mathrm{~g}(19.0 \%)$, menores a $200 \mathrm{~g}(4.8 \%)$, y finalmente los que cosechaban con pesos mayores a $350 \mathrm{~g}(4.8 \%)$.

La trucha entera representa la principal forma de comercialización (66.6\%), seguido por el $14.3 \%$ que es comercializada en plato, el $9.5 \%$ es vendida eviscerada, el $4.8 \%$ como alevín y $4.8 \%$ como filete. El $85.7 \%$ vende su producto en sus mismos centros de producción, mientras el restante lo comercializa en mercados locales. En cuanto al precio, el $80.1 \%$ de los productores comercializa el kilogramo de trucha entre 15 y 25 soles, mientras que el $19.9 \%$ a un precio inferior de 15 soles (1 US $\$=\mathrm{S} / .3 .25$ ).

\section{Literatura Citada}

1. Ayik Ö, Akyurt Ý. 1996. Effects of covering ponds with different materials (earthen, nylon, concrete, gravel) on growth, survival and feed conversion rates in rainbow trout (Oncorhynchus mykiss). Turk J Vet Anim Sci 20: 283-286.

2. Becker JA, Speare DJ, Dohoo IR. 2005. Influence of feeding ratio and size on susceptibility to microsporidial gill disease caused by Loma salmonae in rainbow trout, Oncorhynchus mykiss (Walbaum). J Fish Dis 28: 173-180. doi: 10.1111/j.1365-2761.2005.00614.x 
3. Davidson J, Good C, Welsh C, Summerfelt S. 2011. The effects of ozone and water exchange rates on water quality and rainbow trout Oncorhynchus mykiss performance in replicated water recirculating systems. Aquacult Eng 44: 80-96. doi: 10.1016/ j.aquaeng.-2011.04.001

4. [FAO] Organización de las Naciones Unidas para la Alimentación y la Agricultura. 2016. El estado mundial de la pesca y la acuicultura 2016. Italia, Roma: FAO. [Internet]. Disponible en: http://www.fao.org/3/a-i5555s.pdf

5. [FAO] Organización de las Naciones Unidas para la Alimentación y la Agricultura. 2017. Producción pesquera y acuícola en América Latina y el Caribe. Roma, Italia: FAO. [Internet]. Disponible en: http:// www.fao.org/americas/prioridades/pesca y acuicultura/es/

6. [FONDEPES] Fondo Nacional de Desarrollo Pesquero. 2014. Manual de crianza de trucha en ambientes convencionales. Perú. [Internet]. Disponible en: https://www.fondepes.gob.pe/src/manuales/MANUAL_TRUCHA.pdf

7. García-Mondragón D, GallegoAlarcón I, Espinoza-Ortega A, García-Martínez A, Arriaga-Jordán CM. 2013. Desarrollo de la producción de trucha arcoíris (Oncorhynchus mykiss) en el Centro de México. Rev AquaTIC 38: 46-56.

8. Person-Le Ruyet J, Labbé L, Le Bayon $N$, Sévère A, Le Roux A, Le Delliou $\boldsymbol{H}$, et al. 2008. Combined effects of water quality and stocking density on welfare and growth of rainbow trout (Oncorhynchus mykiss). Aquat Living
Resour 21: 185-195. doi: 10.1051/ alr:2008024

9. [PRODUCE] Ministerio de la Producción. 2017. Situación actual de la acuicultura en el Perú. [Internet]. Disponible en: http://www2.produce.gob.pe/ RepositorioAPS $/ 3 /$ jer/ACUISUBMENU4/boletines/SITUACI\%C3\%93N\%20ACTUAL-\%20DE\%20 LA \% 20 ACUIC-ULTURA \% 20EN\%-20EL\%20PER\%-C3\%9A.pdf

10. [PRODUCE] Ministerio de la Producción. 2016. Anuario estadístico pesquero y acuícola 2016. Lima, Perú. [Internet]. Disponible en: http:// ogeiee.produce.-gob.pe/index.php/ shortcode/oee-documentos-publicaciones/publicaciones-anuales/item/775-anuario-estadistico-pesquero-y-acuicola-2016

11. Ruíz M. 1989. El enfoque de sistemas en la investigación pecuaria y su metodología en América Latina. En: Nolte E, Ruiz M (eds). Ciencias sociales y enfoque de sistemas agropecuarios. Lima: RISPAL. p 9-28.

12. Speare DJ, MacNair N, Hammell KL. 1995. Demonstration of tank effect on growth indices of juvenile rainbow trout (Oncorhynchus mykiss) during an ad libitum feeding trial. Am J Vet Res 56: 1372-1379.

13. Yapuchura A. 2002. Producción y comercialización de truchas en el departamento de Puno y nuevo paradigma de producción. Tesis de Maestría. Lima, Perú: Univ. Nacional Mayor de San Marcos. $164 \mathrm{p}$.

14. Zaldivar R. 1989. Sistemas de producción amazónicos. Primer Informe. Pucallpa: IVITA, UNMSM. CIID. 80 p. 